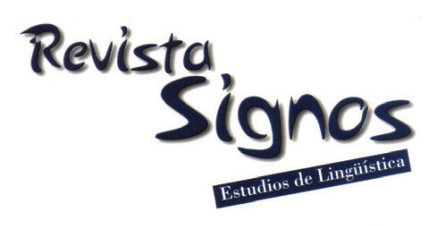

\title{
Género Caso Clínico: Organización retórica de su macromovida Relato del Caso en publicaciones médicas chilenas
}

\author{
Clinical Case Study Genre: Rhetorical organization of the \\ macromove Case Report in chilean medical journals
}

\author{
Gina Burdiles Fernández \\ UNIVERSIDAd CATÓLICA DE LA SANTÍSIMA CONCEPCIÓN \\ CHILE \\ gburdiles@ucsc.cl
}

Recibido: 20/VI/2014 / Aceptado: 23/XII/2015

\section{Resumen}

Uno de los géneros más antiguos del discurso médico es el Caso Clínico, en el que se reporta algún evento médico nuevo o inusual relativo a una enfermedad, sintomatología o tratamiento. Se hace necesario profundizar en el conocimiento de los patrones de regularidad que subyacen al modo en que se organiza retóricamente este género, puesto que posee alto valor pedagógico en la formación profesional y es determinante en el éxito de los jóvenes profesionales de la medicina que comienzan a publicar (Uribarri, 2007; Serrano, 2010). Como parte de un proyecto más amplio, en este artículo, se describe la organización retórica del Relato del Caso, unidad retórica que corresponde a una de las principales macromovidas que configuran al género Caso Clínico (Burdiles, 2012). El propósito de este estudio es determinar, mediante el Análisis del Género (Swales, 1990, 2004), la organización retórica de la macromovida Relato del Caso, a partir de un corpus conformado por 969 textos provenientes de nueve especialidades médicas (Corpus CCM-2009). Según los resultados, esta macromovida tiene una organización retórica constituida por tres movidas y ocho pasos retóricos. Se observó, por un lado, que este patrón se manifiesta con bastante regularidad en las nueve disciplinas médicas, lo que comprobaría la existencia de un sistema común de convenciones retóricas manifestadas en la escritura del género. Por otro lado, se constató que dichas convenciones presentan algunas variaciones asociadas a la naturaleza de las distintas especialidades médicas.

Palabras Clave: Organización retórica, movida retórica, caso clínico, discurso de la medicina, género discursivo. 


\begin{abstract}
The Clinical Case Study is one of the oldest medical discursive genres where a new medical or unusual illness-related event, symptomatology or treatment is reported. It is necessary to deepen the knowledge of the patterns of regularity that underlie the way this genre is rhetorically organized as it carries a high pedagogical value in vocational training and in determining the success of young medical professionals who start publishing (Uribarri, 2007; Serrano, 2010). As part of a wider project, this article describes the rhetorical organization of the Case Report, a unit that corresponds to one of the main macromoves that shape the Clinical Case Study genre (Burdiles, 2012). The purpose of this study is to determine, by means of Genre Analysis (Swales, 1990, 2004), the rhetorical organization of the macromove Case Report of a corpus of 969 texts from nine medical specialties (Corpus CCM-2009). According to the results, this macromove has a rhetorical organization made up of three moves and eight rhetorical steps. It was observed, on one hand, that this pattern appears fairly regularly in the nine medical specialties, which would prove the existence of a common system of rhetorical conventions expressed in genre writing. On the other hand, it was found that these conventions have some variations associated with the nature of the various medical specialties.
\end{abstract}

Key Words: Rhetorical organization, rhetorical move, case report, discourse of medicine, discursive genre.

\title{
INTRODUCCIÓN
}

Las publicaciones científicas periódicas son el principal medio de comunicación para las comunidades científicas. En el caso particular de la Medicina, Goic (2002) sostiene que junto con promover el intercambio científico, todo lo que se publica contribuye, además, a los avances educativos en la disciplina. Existe un variado conjunto de géneros que resultan de la actividad científica y profesional de esta comunidad discursiva particular y que son publicados en revistas especializadas de medicina. Dentro de ellos, el Caso Clínico es un género que tiene gran reconocimiento por parte de la comunidad discursiva en que circula, debido a su rol en el estímulo de líneas investigativas y en el desarrollo acumulativo del conocimiento disciplinar; además, por su incidencia en el proceso educativo en la formación profesional.

A este respecto, se sabe, además, que uno de los aspectos determinantes en el éxito de los jóvenes profesionales de la Medicina que comienzan a publicar es el conocimiento de los patrones de las regularidades que subyacen al modo en que se organiza un género (Nwogu, 1995, 1997; Uribarri, 2007). Sin embargo, la distinción entre los diversos géneros de esta disciplina no está exenta de complejidad, dado que los géneros no son constructos fijos ni homogéneos; al contrario, se caracterizan por ser dinámicos y por poseer rasgos que, aun estando sujetos a las convenciones y al conocimiento de la comunidad discursiva donde circulan, están continuamente desarrollándose, cambiando y dando pie al surgimiento de nuevas pautas de textualización (Swales, 1990, 2004; Bhatia, 1993, 2004; entre otros). Por su dinamismo, la forma que los identifica puede hasta ser híbrida ya que los géneros pueden presentar 
variaciones disciplinarias, especialmente en contextos académicos (Bazerman \& Paradis, 1991; Parodi, 2008a). En atención a esto, surge la pregunta de cuánto se conoce empíricamente acerca de la organización retórica del Caso Clínico.

El discurso de la Medicina ha sido ampliamente estudiado, particularmente en lengua inglesa y preferentemente centrándose en el género Artículo de Investigación Científica (Atkinson, 1992, 1995, 2004; Skelton, 1994, 1997; Nwogu, 1997; Varttala, 2001; Alcaraz; 2003; Hiltunen, 2006; ElMalik \& Nesi, 2008; Li \& Ge, 2009). El Caso Clínico de Medicina, en cambio, ha sido menos estudiado en inglés y muy escasamente en lengua española (Adams-Smith, 1984; Salager-Meyer, 1994; Taavitsainen \& Pahta, 2000; Salager-Meyer \& Alcaraz, 2003; Alcaraz \& Salager-Meyer, 2003; Carey, 2006a; Morales, 2010).

Este artículo presenta parte de una investigación mayor que tuvo como fin profundizar en el conocimiento de la organización retórica del Caso Clínico de Medicina. En dicha investigación, Burdiles (2012) comprobó, a partir del análisis de un amplio corpus de textos completos, que la macromovida Relato del Caso, la que es objeto de estudio en el presente artículo, es la más destacada del género Caso Clínico. De acuerdo con la literatura de la Medicina, es la unidad más importante en la organización retórica del Caso Clínico (Uribarri, 2007). Jenicek (2001: 105) la considera "the core of the message" y corresponde exactamente a la presentación detallada del caso, donde se menciona el estado inicial de un paciente y su evolución hasta los resultados finales. Siguiendo la metodología del Análisis del Género y a partir del examen de un corpus conformado por 969 textos, provenientes de nueve especialidades médicas (Corpus CCM-2009), en este estudio se describe la organización retórica prototípica de la macromovida Relato del Caso y se compara su realización en nueve especialidades médicas comprendidas en el corpus.

En lo que sigue, se comienza abordando los conceptos clave del marco referencial y luego se describe el marco metodológico de la investigación atendiendo a sus objetivos, su enfoque, su diseño, el corpus y los procedimientos de análisis de los datos. A ello, le sigue la entrega de los resultados del análisis que permitió configurar la organización retórica de la macromovida Relato del Caso y la comparación de la misma a través de las nueve especialidades médicas contempladas en el corpus estudiado. Finalmente, se presentan las conclusiones a que condujo el estudio.

\section{Marco de referencia}

\subsection{El Análisis del Género y la organización retórica}

Para Swales (1990) y Bhatia (1993), los géneros son tipos de eventos comunicativos que poseen rasgos convencionales recurrentes conocidos por la comunidad de discurso que los usa y que se distinguen por su propósito comunicativo. Según Swales (1990), los propósitos comunicativos constituyen el criterio fundamental para definir 
un género, para determinar sus alcances, para configurar su estructura esquemática y para guiar las elecciones de su contenido y estilo. Bathia (1993) aporta que el conjunto de estos propósitos se entiende también compartido por la comunidad discursiva y configura al género dotándolo de una estructura interna.

El Análisis del Género estudia los rasgos retórico-discursivos de los textos que constituyen un género, puesto que comparten determinados patrones comunicativos y dado que los mismos son conocidos por los miembros de la comunidad discursiva donde ese género circula. Originalmente, fue propuesto por Swales (1981, 1990, 2004), con miras a implementar mejoras en los procesos de enseñanza de lengua con propósitos específicos, y apuntaba a describir la organización de una sección del género Artículo de Investigación Científica publicado en inglés. La creciente valoración del Análisis del Género como metodología útil para dar cuenta de sus patrones organizacionales ha dado pie en los últimos treinta años a numerosas investigaciones que han descrito la organización retórica de numerosos géneros. Entre otros, debe mencionarse los trabajos de Bhatia (1993, 2004); Kanoksilapatham (2005, 2007a, 2007b, 2007c); Biber, Connor y Upton (2007); Samraj (2002); Parodi (2008a, 2008b).

La organización retórica es la representación de la estructura funcional de un género, a partir de la sistematización de sus unidades y subunidades retóricodiscursivas. La unidad discursiva básica para desarrollar este análisis es la 'movida', definida por Swales (2004) como una unidad discursiva o retórica que realiza una función comunicativa coherente, en un discurso oral o escrito. De manera que el análisis de género basado en movidas:

"(...) represents academic research articles in terms of hierarchically organized text made up of distinct sections; each section can be subdivided into moves, and each move can be broken down into steps" (Kanoksilapatham, 2005: 271).

Una movida, en tanto, es, a juicio de Holmes (1997), un segmento del texto configurado, estructurado y determinado por alguna función comunicativa específica. Cada movida no solo tiene su propia finalidad, sino que también contribuye a la comunicación del propósito retórico global del género (Connor, Davis \& De Rycker, 1995; Kanoksilapatham, 2007a). Tal como la identificación de las movidas y pasos obedece a niveles de abstracción diferentes, en el caso de los propósitos asociados, es posible establecer una jerarquía similar. En atención a esto, Parodi (2008a) propone el concepto de Macromovida, unidad discursiva mayor a la que se asocia un Macropropósito, el que puede ser entendido como propósito comunicativo del género (Swales, 1990, 2004; Bhatia, 1993, 2004; Kanoksilapatham, 2007c). De este modo, el conjunto de unidades retóricas (macromovidas, movidas y pasos), asociadas a la realización de propósitos comunicativos específicos, configura la organización, más o menos convencionalizada, que caracteriza a un género. 


\subsection{El Caso Clínico en el estudio del discurso especializado de la Medicina}

El quehacer académico y profesional de la Medicina es comunicado en géneros de diversa complejidad y especialización, entre los que destacan los Resúmenes, los Artículos de Investigación Científica, los Artículos de Revisión, los Editoriales y los Casos Clínicos. Una revisión de la situación actual sobre este campo revela que las investigaciones que han estudiado los patrones organizacionales de los géneros del discurso médico se han centrado principalmente en el Artículo de Investigación Científica (Swales, 1981; Crookes, 1986; Atkinson, 1992; Skelton, 1994, 1997; Nwogu, 1997; El Malik \& Nesi, 2008; Li \& Ge, 2009). La revisión de la literatura evidencia que lo que se conoce a este respecto sobre el Caso Clínico es poco y que ha sido escasamente estudiado en español. Algunas descripciones de carácter prescriptivo provienen principalmente de la preocupación de la propia comunidad médica por optimizar la difusión de la producción científica en esta disciplina (Vandenbroucke, 2001; Valladolid \& Pérez, 2005; Cohen, 2006; Bart \& Green, 2006; Carey, 2006a, 2006b; Jefferson, 2008). Otra fuente de conocimiento sobre este género proviene, en menor medida, de algunas investigaciones realizadas desde distintas perspectivas lingüísticas (Adams-Smith, 1984; Salager-Meyer, 1992, 1994; Taavitsainen \& Pahta, 2000; Salager-Meyer \& Alcaraz, 2003; Alcaraz \& Salager-Meyer, 2003; Oliver, 2004; Morales, Cassany, Marín \& González, 2007; Morales, 2010).

El Caso Clínico es un género médico escrito en el ámbito de la actividad profesional e investigativa, que permite el intercambio comunicativo -preferentemente- entre interlocutores que están en relación simétrica, esto es, que mantienen una comunicación de experto a experto (Gotti, 2003). Para Adams-Smith (1984), los Casos Clínicos son la más breve y simple categoría de artículo en Medicina y se basan en el reporte médico de un caso o un pequeño grupo de casos inusuales. Son definidos como:

"Brief reports describing an isolated clinical case or a small number of cases. They may describe new or uncommon diagnoses, unusual outcomes or prognosis, new or infrequently used therapies and side effects of therapy not usually discovered in clinical trials" (Khan \& Thompson, 2002: 849).

Dado que describen algún evento médico (o un par de eventos) que ofrece a la comunidad médica un aspecto nuevo o inusual relativo a una enfermedad, a una sintomatología o a un tratamiento, tienen el valor de proponer las primeras líneas de evidencia acerca de un evento centinela, es decir, un suceso no deseado e imprevisto que tienen lugar durante la asistencia sanitaria, y que conlleva consecuencias negativas para los pacientes y para las instituciones sanitarias (Xunta de Galicia, 2013). De este modo, el Caso Clínico contribuye al conocimiento médico, en la medida en que presentan aspectos nuevos o instructivos de una enfermedad determinada (Khan \& 
Thompson, 2002; Reyes \& Llanos, 2002; Pertuzé, 2006; Berkenkotter, 2008). Además de circular en contextos de comunicación especializada entre pares, este género es considerado esencial en la enseñanza médica, tanto para los estudiantes de pre y postgrado, como para los residentes y médicos especialistas (Gérvas, Pérez, Cuñat \& Martínez, 2002; Serrano, 2010). En ese sentido, Jenicek (2001) señala que tiene finalidades profesionales y científicas, y subraya que es una forma de comunicación que posee sus reglas específicas.

\subsection{El Relato del Caso, una macromovida del Caso Clínico}

En el siglo XVII, a raíz de la determinación de modelos de comunicación escrita establecidos para la Philosophical Transactions of the Royal Society of London, comienzan a establecerse algunas de las pautas de textualización para las publicaciones del área médica (Taavitsainen \& Pahta, 2000). Mucho más tarde, a partir del 1972, se propone para las publicaciones médicas, en general, el formato IMRD (Introducción, Métodos, Resultados y Discusión), formato al que el Caso Clínico no se ajusta con comodidad:

"(...) the usual "IMRAD” format (Introduction, Methods, Results, and Discussion) that one sees in reports of clinical research might not always be appropriate for case reports and case series" (Vandenbroucke, 2001: 333).

Jenicek (2001), desde la comunidad médica, sugiere que el Caso Clínico debería estar constituido por cinco secciones: resumen, introducción, presentación del caso, discusión-conclusión y referencias. Sin embargo, una revisión de las actuales líneas editoriales de las publicaciones médicas chilenas permite constatar que no hay sistematicidad en proponer un formato específico para este género. En consecuencia, quienes escriben Casos Clínicos deben ajustarse a las pautas de publicación del Artículo de Investigación Científica de Medicina, definidas internacionalmente por los estándares del Estilo de Vancouver (Valladolid \& Pérez, 2005).

En relación con la configuración discursiva del Caso Clínico descrita desde una perspectiva lingüística, en 1984, Adams-Smith esbozó una estructura discursiva del Caso Clínico en inglés, a partir del examen de seis ejemplares. Observó que comienzan con una breve introducción que, a su juicio, funciona como un abstract, luego presentan el reporte del caso propiamente tal y finalizan por una sección de comentario o discusión. Diez años más tarde, Salager-Meyer (1994), a partir del examen de 10 Casos Clínicos, también en inglés, y con la finalidad de observar los dispositivos de mitigación discursiva en distintas secciones retóricas, asume que el patrón estructural de este género posee tres constituyentes: "a short Introduction, a detailed Case Report, and a brief Comment (sometimes called Discussion) section" (Salager-Meyer, 1994: 153). Por su parte, Taavitsainen y Pahta (2000), quienes dan una mirada diacrónica al género en lengua inglesa, proponen que un Caso Clínico típico comienza por declarar la razón que motiva el reporte del caso, luego da lugar a 
una sección frecuentemente titulada case report y posteriormente da paso a una parte final que puede llamarse comment. Una década más tarde, Morales (2010), basado en el estudio de un corpus de 40 casos clínicos de odontología en español, reporta que sus resultados no revelan una estructura retórica uniforme. Sin embargo, destaca una tendencia a mostrar el siguiente formato o estructura: Introducción, Presentación del caso y Discusión/Conclusión (Morales et al., 2007). El estudio de Morales (2010) adelanta en la exploración de las movidas retóricas de las tres secciones que conformarían el Caso Clínico odontológico en español; sin embargo, mantiene -al igual que los otros estudios recién reseñados- una visión estructural que limita cualquier explicación acerca de los distintos propósitos comunicativos perseguidos por las unidades retóricas que organizarían al género. Por otra parte, las descripciones reseñadas no se basaron en el estudio de corpus amplios.

Burdiles (2012) desarrolló una investigación doctoral que pretendió corroborar, sistematizar y profundizar las descripciones existentes con el aporte de datos provenientes de un estudio empírico en el que trabajó con muestras mayores de textos y en el que, además, consideró los textos de manera íntegra. A partir del supuesto de que los textos que pertenecen a un género exhiben una organización funcional más o menos estable, el estudio de Burdiles (2012) describió, en términos de sus movidas retóricas constitutivas, la organización retórica del género Caso Clínico, a partir del análisis de un amplio corpus, conformado por 969 textos provenientes de nueve especialidades médicas (Corpus CCM-2009).

Los datos provenientes de este análisis del Corpus CCM-2009 permitieron determinar la presencia regular de las siguientes cuatro macromovidas retóricas y sus correspondientes propósitos comunicativos.

Tabla 1. Organización Retórica del género Caso Clínico (Burdiles, 2012).

\begin{tabular}{|l|l|}
\hline \multicolumn{2}{|c|}{ Organización Retórica del género Caso Clínico } \\
\hline \multicolumn{1}{|c|}{ Macromovida } & \multicolumn{1}{c|}{ Propósito Comunicativo } \\
\hline 1. Preámbulo & Establecer el marco general donde se sitúa el problema que se reporta \\
\hline 2. Relato del caso & Narrar el desarrollo del caso \\
\hline 3. Análisis & Examinar los procesos del caso a la luz de la literatura \\
\hline 4. Cierre & Cerrar el reporte del caso, enfatizando sus aportes \\
\hline
\end{tabular}

Esta descripción difiere de la conformación tripartita del Caso Clínico planteada por Adams-Smith (1984), Salager-Meyer (1994), Taavitsainen y Pahta (2000), y Morales (2010). A juicio de Burdiles (2012), después de la macromovida Preámbulo, esto es, después de haber establecido el marco general donde se sitúa el problema médico que se reporta, y antes de la macromovida Cierre, cuyo propósito es dar por cerrado el reporte del caso, el género Caso Clínico responde a otros dos propósitos distintos, pero de similar importancia. Por un lado, en el Relato del Caso, se narra el desarrollo de la historia que constituye el ‘caso' y, por otro, en el Análisis, se realiza un examen posterior de los procesos descritos a la luz de la literatura revisada. 
Según Burdiles (2012), de las cuatro macromovidas mediante las cuales se realiza el género Caso Clínico de Medicina, el Relato del Caso es la más característica de su organización retórica, puesto que es la única macromovida que -invariablementeocurre en todos los textos del corpus. Sin lugar a dudas, esta observación permite corroborar con evidencia empírica lo sostenido por algunos autores que asignan una importancia central a la descripción de caso en el desarrollo de los Casos Clínicos (Jenicek, 2001; Uribarri, 2007).

\section{El estudio}

\subsection{Objetivos}

Este estudio se propone, en primer término, identificar, mediante el Análisis del Género, un conjunto de unidades retóricas (movidas y pasos) que permitan caracterizar la organización retórica de la macromovida Relato del Caso, en términos de los propósitos comunicativos de dichas movidas y pasos; en segundo término, se pretende comparar su organización retórica a través de las nueve especialidades médicas comprendidas en el Corpus CCM-2009.

\subsection{Tipo de investigación}

La presente investigación se enmarca en un enfoque mixto, el que implica la recolección, el análisis y la vinculación de datos de naturaleza cuantitativa y cualitativa (Tashakkori \& Teddlie, 2003; Mertens, 2005). Esta secuencia es útil cuando se desea primero explorar el planteamiento de un problema para posteriormente expandir su entendimiento en una muestra mayor (Hernández, Fernández \& Baptista, 2010), además, es un enfoque sugerido para el análisis de discurso especializado (Biber, 1988; Alcaraz \& Salager-Meyer, 2003; Biber et al., 2007; Parodi, 2008a, 2008b).

En cuanto al modelo general de diseño, se utilizó un modelo no experimental, transeccional y de ejecución secuencial. La siguiente tabla presenta las principales etapas en que se desarrolló el estudio.

Tabla 2. Etapas del estudio.

\begin{tabular}{|l|}
\hline 1. Conformación del Corpus CCM-2009. \\
\hline 2. Propuesta preliminar de la organización retórica del Relato del Caso, a partir del examen de \\
un microcorpus: generación de un listado de unidades retóricas. \\
\hline 3. Validación de la propuesta preliminar por medio de cinco pares expertos. \\
\hline 4. Revisión y ajustes de la propuesta preliminar. \\
\hline 5. Aplicación de la propuesta: identificación y registro de las frecuencias de aparición de las \\
unidades retóricas del Relato del Caso en todo el Corpus CCM-2009. \\
\hline 6. Establecimiento y descripción de un patrón definitivo de la organización retórica del Relato \\
del Caso, a partir de las frecuencias registradas. En esta fase se determinó qué unidades son \\
centrales para la caracterización de la macromovida y cuáles son periféricas. \\
\hline 7. Examen de la organización retórica de la macromovida Relato del Caso a través de las nueve \\
especialidades médicas. \\
\hline
\end{tabular}


En relación con el alcance de este estudio, esta es una investigación descriptiva, que pretende especificar los rasgos de un fenómeno y sus tendencias. Las variables que fueron objeto de observación son las siguientes:

- Organización retórica: estructura funcional de un género, a partir de la sistematización de sus unidades y subunidades retórico-discursivas.

- Movida retórica: unidad retórico-discursiva que realiza un propósito comunicativo y que es susceptible de ser identificada a partir de pistas textuales. En conformidad con el grado de abstracción de dicha unidad, se hablará de macromovida, movida o paso retórico.

- Propósito comunicativo: “objetivo último para el cual un género discursivo es utilizado en un intercambio comunicativo" (Parodi, Venegas, Ibáñez \& Gutiérrez, 2008: 47). Al igual que las movidas, obedece a niveles de abstracción diferentes.

\subsection{El Corpus CCM-2OO9}

El corpus con que se trabajó estuvo conformado por 969 textos en archivo digital, correspondientes a la totalidad de artículos identificados como Casos Clínicos, publicados en nueve revistas chilenas de Medicina de diversas especialidades, en la década 1999-2008 (ver Tabla 3). Todas ellas están indexadas en SciELO y clasificadas por tema en Ciencias de la Salud. Además, a juicio de expertos en el campo de las publicaciones médicas nacionales, cuentan con un reconocido prestigio por parte de la comunidad médica. Las publicaciones se caracterizan por estar en español, tener diferente periodicidad, contar con el respaldo de diferentes colectivos profesionales, publicar artículos de distintas especialidades médicas, someter sus artículos al arbitraje de pares antes de la publicación y, lo más importante, por incluir el Caso Clínico entre los artículos publicados en el periodo abarcado por este estudio.

En relación con el tamaño del corpus, la Tabla 3, indica el número de textos provenientes de cada publicación y el número de palabras que lo constituyen; asimismo, se señala el promedio de palabras que el Caso Clínico posee en cada especialidad. En la segunda columna se indican los códigos usados para identificar cada especialidad. 
Tabla 3. Cantidad de textos y de palabras por publicación fuente.

\begin{tabular}{|l|c|c|c|c|}
\hline \multicolumn{1}{|c|}{ Revista } & Código & $\begin{array}{c}\mathbf{N}^{\mathbf{o}} \text { de } \\
\text { textos }\end{array}$ & $\begin{array}{c}\mathbf{N}^{\mathbf{o}} \text { de } \\
\text { palabras }\end{array}$ & $\begin{array}{c}\text { Promedio } \\
\text { de } \\
\text { palabras }\end{array}$ \\
\hline Parasitología Latinoamericana & $\mathrm{Pa}$ & 8 & 15.415 & 1.926 \\
\hline Revista Chilena de Neuro-Psiquiatría & $\mathrm{NP}$ & 20 & 45.877 & 2.293 \\
\hline Revista Chilena de Enfermedades Respiratorias & $\mathrm{ER}$ & 31 & 54.523 & 1.758 \\
\hline Revista de Otorrinolaringología y Cirugía de Cabeza y Cuello & OCCC & 52 & 96.506 & 1.855 \\
\hline Revista Chilena de Infectología & $\mathrm{In}$ & 73 & 138.269 & 1.894 \\
\hline Revista Chilena de Pediatría & $\mathrm{Pe}$ & 108 & 192.849 & 1.785 \\
\hline Revista Chilena de Obstetricia y Ginecología & $\mathrm{OG}$ & 168 & 253.293 & 1.507 \\
\hline Revista Chilena de Cirugía & $\mathrm{Ci}$ & 170 & 222.381 & 1.308 \\
\hline Revista Médica de Chile & $\mathrm{MI}$ & 339 & 525.746 & 1.550 \\
\hline Totales & & 969 & 1.544 .859 & 1.595 \\
\hline
\end{tabular}

Estos datos revelan que el Caso Clínico es un género cuyos textos se caracterizan por tener una extensión relativamente corta, con un promedio de 1.595 palabras por texto.

\subsection{Procedimiento de análisis de los datos}

Para identificar las movidas y pasos que conforman la organización retórica de la macromovida Relato del Caso y determinar sus respectivos propósitos comunicativos, se siguieron las orientaciones de Biber et al. 2007 y Kanoksilapatham (2007a, 2007b) en lo referido a los pasos analíticos para lograr una descripción de estructura de discurso basada en corpus; asimismo, se siguió el trayecto metodológico descrito por Parodi (2008a, 2008b) para dar cuenta de la organización retórica de un género académico.

Como ya se adelantó en la Tabla 2, el análisis de las movidas retóricas consideró, primero, el desarrollo de un marco analítico funcional que permitiera la generación de una propuesta preliminar sobre cómo se configuraban las unidades retóricas del Relato del Caso. En esta fase, se segmentaron los textos en unidades funcionales discursivas menores, conocidas como 'movidas', esto es, unidades retóricas susceptibles de ser identificadas en relación con la realización de un propósito comunicativo (Swales, 1990; Kanoksilapatham, 2007b). La propuesta preliminar de análisis se validó mediante consulta a cinco pares expertos en análisis del discurso y en Medicina, a quienes se les consultó por la identificación y nominación de las unidades retóricas susceptibles de ser distinguidas en un microcorpus dado. Las categorías que presentaron un grado de acuerdo con promedio superior a $80 \%$, se consideraron validadas y, en términos generales, no fueron modificadas. En cambio, las categorías que presentaban un grado de acuerdo entre los jueces con un promedio inferior al $80 \%$, sufrieron alguna modificación.

Posteriormente, se aplicó la propuesta analítica validada a los textos que conformaban el corpus, y se registraron las frecuencias de aparición de las unidades y 
subunidades observadas en el corpus. El propósito de esta cuantificación fue determinar cuáles movidas y pasos propuestos son aquellos que definen un patrón prototípico de la organización retórica de la macromovida Relato del Caso. Son variados los criterios para decidir el índice de frecuencia a partir del cual se determina la estabilidad de la organización retórica y la distinción entre las unidades retóricas que pueden considerarse centrales o periféricas (Kanoksilapatham, 2007a; Parodi, 2008b; Ibáñez, 2008; Venegas, 2014; Parodi, Boudón \& Julio, 2014); tradicionalmente, el criterio de puntaje de corte adoptado para decidir si una movida es opcional u obligatoria es de 60\% de frecuencia de ocurrencia. Para analizar los datos cuantitativos, se usaron las frecuencias absolutas y relativas.

Para identificar y describir la organización retórica, se empleó una metodología que se sitúa entre los enfoques denominados descendentes y ascendentes, en tanto se complementan los enfoques deductivo e inductivo. Esto quiere decir que no predominó un criterio apriorístico, sino que se comparó los conocimientos previos de la analista y de los expertos consultados en el proceso de validación con las categorías que emergían de los textos analizados. De este modo, en los criterios de segmentación y asignación de propósitos comunicativos, confluyeron los siguientes factores: los conocimientos previos de la investigadora como profesora de lenguas, las prescripciones de la literatura médica para la publicación de Casos Clínicos (artículos e instrucciones para los autores de las revistas especializadas), las descripciones de la literatura para este género en inglés, los datos empíricos provenientes de la observación de varios microcorpus de Relato del Caso en Casos Clínicos, y -como se ha mencionado- el juicio de expertos consultados para el diseño de la propuesta de organización retórica.

\section{Análisis de resultados}

En conformidad con los objetivos de este estudio, en lo que sigue se entregan, en primer lugar, los resultados sobre las unidades retóricas (movidas y pasos) que conforman la organización retórica de la macromovida Relato del Caso, en términos de sus propósitos comunicativos; luego, se compara la misma a través de las especialidades médicas comprendidas en el corpus en estudio.

\subsection{Organización retórica de la macromovida Relato del Caso}

\subsubsection{Macromovida Relato del Caso: Sus movidas y pasos}

Después del examen preliminar del corpus y de la consulta a juicio de expertos, se identificó el siguiente conjunto de unidades retóricas en la macromovida Relato del Caso. 


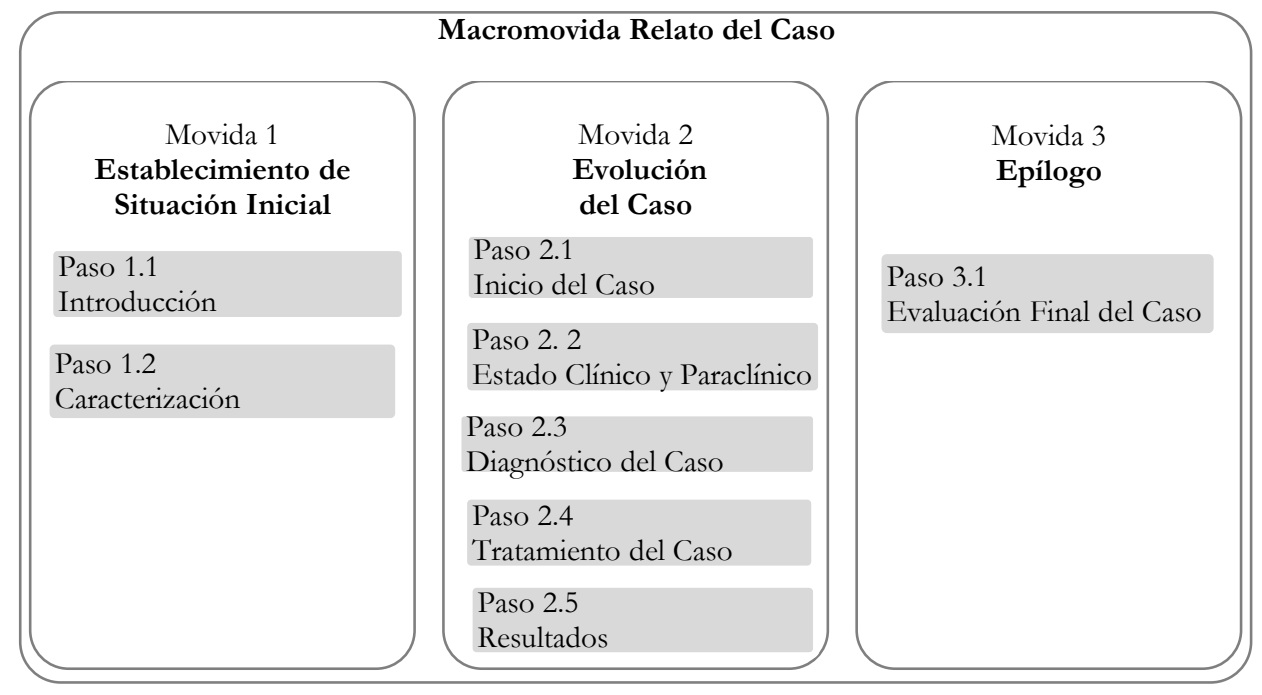

Figura 1. Movidas y pasos de la macromovida Relato del Caso.

Como lo indica la Figura, la organización retórica quedó conformada por tres movidas y ocho pasos retóricos. La movida 2 es la que presenta mayor complejidad, puesto que la consecución de su propósito comunicativo se realiza mediante cinco pasos retóricos. Más adelante nos referiremos en detalle a los propósitos comunicativos de estas unidades retóricas.

La frecuencia de ocurrencia de estas movidas y pasos en la totalidad de corpus está detallada en el Gráfico 1.

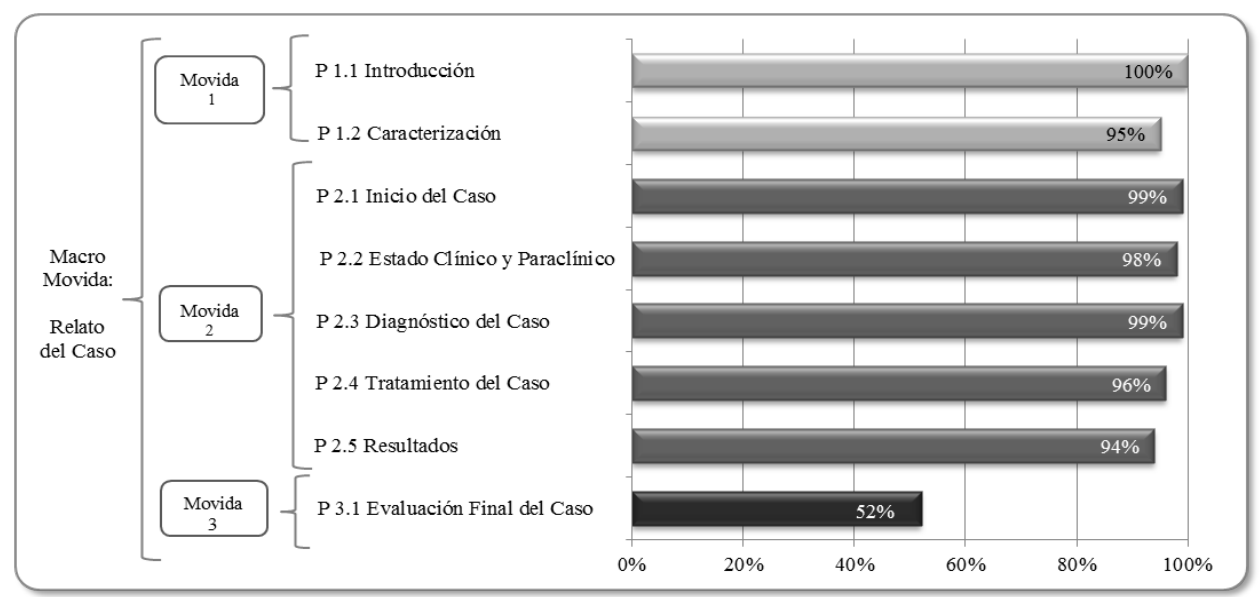

Gráfico 1. Ocurrencia de movidas y pasos en macromovida Relato del Caso.

Resulta evidente, al observar el Gráfico 1, que todos los pasos de las movidas 1 y 2 son altamente recurrentes, es decir, se detectan en más del $90 \%$ de los textos del 
corpus. Esto indica que la macromovida Relato del Caso no podría prescindir de alguno de ellos para cumplir sus propósitos comunicativos, esto es, presentar al paciente, luego describir sus condiciones al momento de declararse el caso y después dar cuenta de todo el proceso posterior de observación e intervención médica. Algo distinto ocurre con la movida 3 y su paso retórico Evaluación Final del Caso, cuya frecuencia de $52 \%$ indica que se trata de una movida opcional para el género Caso Clínico. Casi en la mitad de los textos analizados no aparece el epílogo del relato, es decir, no fue incorporada información acerca de qué ocurrió con la historia relatada, más allá de sus resultados inmediatos. No obstante la baja frecuencia y dado que este estudio, además de proponer un caracterización de la organización retórica de la macromovida en estudio, se propone indagar la variación de la misma en las especialidades médicas, se tomó la decisión de considerar también la movida 3 en el análisis.

\subsubsection{Macromovida Relato del Caso: Sus propósitos comunicativos}

Como lo mostró la Figura 1, el Relato del Caso se realiza mediante tres movidas y ocho pasos retóricos. En la Tabla 4, se explicitan cuáles son los propósitos comunicativos que, a partir del análisis del corpus CCM-2009, se determinaron para dichas movidas y pasos.

Tabla 4. Movidas, pasos y propósitos comunicativos del Relato del Caso.

\begin{tabular}{|c|c|}
\hline \multicolumn{2}{|c|}{ Organización Retórica de macromovida Relato del Caso } \\
\hline Movida/Paso & Propósito Comunicativo \\
\hline Movida 1 Establecimiento de Situación Inicial & Presentar al paciente \\
\hline Paso 1.1 Introducción & Instalar en escena al paciente \\
\hline Paso 1.2 Caracterización & Proveer de un contexto \\
\hline Movida 2 Evolución del Caso & Exponer las fases del proceso que experimenta el caso \\
\hline Paso 2.1 Inicio del Caso & Comenzar el relato del caso \\
\hline Paso 2.2 Estado Clínico y Paraclínico & Describir cuadro previo al diagnóstico del caso \\
\hline Paso 2.3 Diagnóstico del Caso & Informar proceso de determinación del diagnóstico \\
\hline Paso 2.4 Tratamiento del Caso & Dar cuenta del desarrollo del tratamiento aplicado \\
\hline Paso 2.5 Resultados & Reportar desenlace del relato \\
\hline Movida 3 Epílogo & Finalizar el relato \\
\hline Paso 3.1 Evaluación Final del Caso & Referir el epílogo del relato \\
\hline
\end{tabular}

Los propósitos de las movidas y pasos permiten concordar con Atkinson (1995) y Morales (2010) en cuanto a que el reporte del suceso clínico llamado Caso Clínico se realiza a través de un complejo de modalidades descriptiva y narrativa. En términos generales, la macromovida Relato del Caso procede describiendo una serie de estados y acciones que dan cuenta de la existencia de un sujeto, el paciente, del proceso de su enfermedad y de las acciones y efectos del diagnóstico y tratamiento seguido. En este sentido, podemos afirmar que la serie de movidas 1, 2 y 3 manifiesta un predominio de la modalidad narrativa, en tanto presentan los constituyentes que debe tener un relato (Brémond, 1966; Adam, 1992). Al igual que en una narración, en esta 
macromovida se presenta una sucesión de acontecimientos; una unidad temática articulada en torno a un actor-sujeto (el paciente); una serie de acciones integradas; presencia de la causalidad narrativa de una puesta en intriga (encadenamiento cronológico por el orden causal) y una evaluación final.

Sin embargo, al interior de la movida 2 Evolución del Caso, algunos pasos retóricos presentan un predominio de la modalidad descriptiva. La movida 2, como puede verse en la Tabla 4, destaca, porque su propósito de exponer las fases del proceso que va experimentando el caso se realiza mediante cinco pasos, con distintos pero complementarios propósitos retóricos. La exposición de las fases del proceso tiene gran valor descriptivo, puesto que se deben entregar detalles que dejen registro de la evolución del paciente. En este sentido, los resultados permiten de algún modo diferir de lo planteado por Uribarri (2007), quien le atribuye al género Caso Clínico una naturaleza eminentemente descriptiva. Sin embargo, se debe concordar con esta autora en que esta modalidad discursiva efectivamente predomina en los pasos de la movida Evolución del Caso, en especial en el paso 2.2 Estado Clínico y Paraclínico. Dicho de otro modo, es en esta movida 2 del Relato del Caso que se revela el carácter escópico del discurso del Caso Clínico, puesto que en ella, más específicamente en sus pasajes descriptivos, entrega mucha información de manera tributaria a la visión y, de ese modo, ofrece al lector (experto o principante) una representación sensible o intelectual de algo que, aunque no está a la vista, de igual modo está allí, mediado por la escritura.

\subsubsection{Dinamicidad al interior de la movida 2 Evolución del Caso}

Con frecuencia, la organización discursiva de la movida 2 Evolución del Caso no corresponde a la descripción de un objeto estático. Jenicek (2001) ya había advertido este dinamismo en el género Caso Clínico, dinamismo que se observa en la recursividad de los pasos que configuran esta movida.

Como se ha visto, esta movida está constituida por una serie de cinco pasos, los cuales conforman una secuencia que frecuentemente puede reiniciarse en cualquier momento, esto quiere decir que, terminado un ciclo, este puede repetirse, tal como lo grafica la Figura 2. 


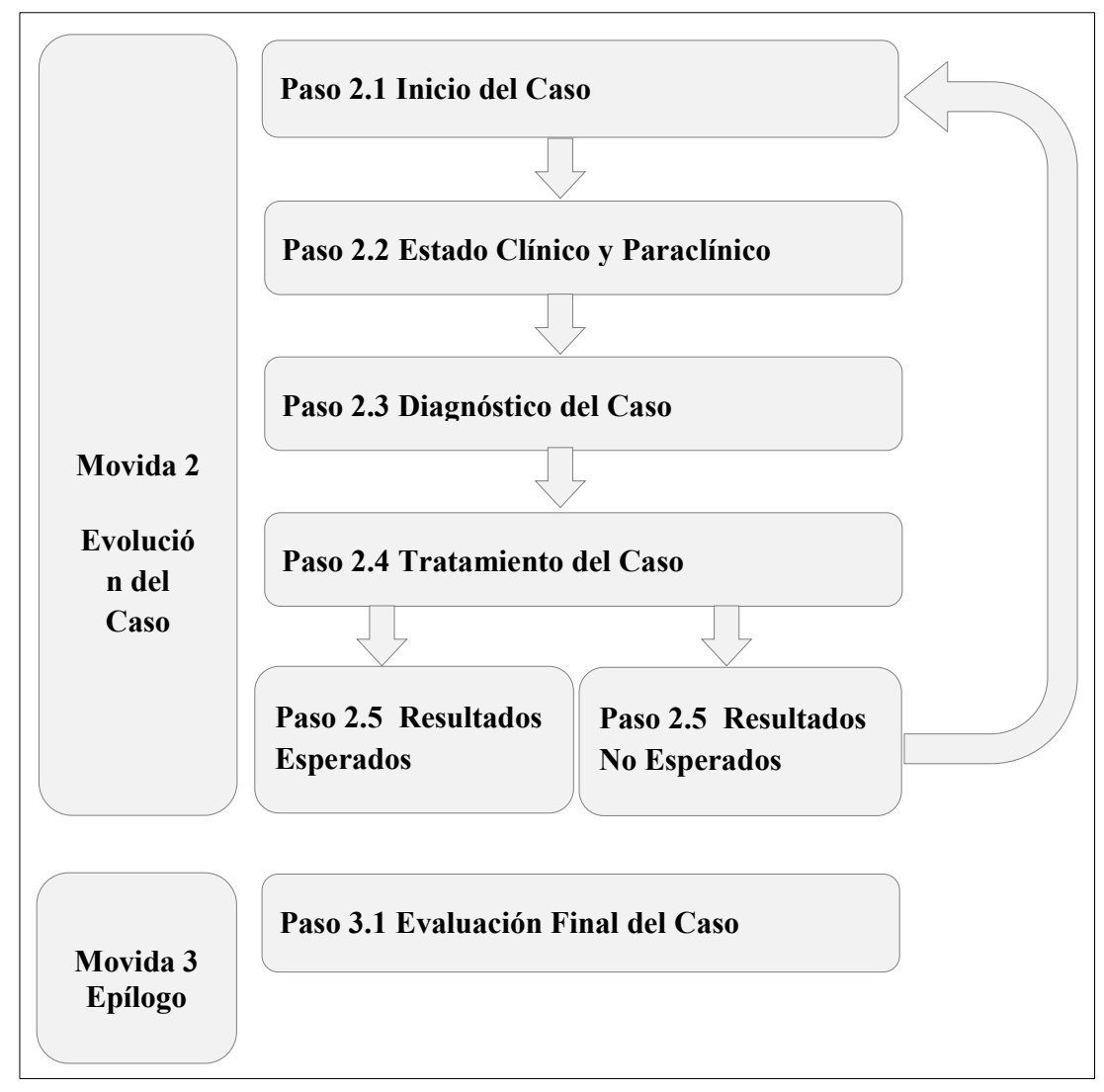

Figura 2. Dinamicidad de la movida 2 Evolución del Caso.

Como muestra la Figura 2, la Evolución del Caso se realiza mediante los pasos que dan cuenta del inicio de un proceso, en un punto de partida específico (Paso 2.1). Luego, se describe el cuadro clínico y paraclínico del paciente, se indica el diagnóstico (que puede ser provisional), después se describe el proceso de tratamiento y enseguida, sus resultados. Sin embargo, desde ese punto, la organización retórica ofrece dos caminos. Si los resultados son los esperados, se cierra el proceso en la movida 3, con la evaluación final del caso. En otras ocasiones, si el diagnóstico presuntivo y el tratamiento no conducen a los resultados esperados, entonces el proceso se reinicia en la descripción de un nuevo estado, ofrecida por el paso 2.2, Estado Clínico y Paraclínico. En síntesis, la organización de la movida Evolución del Caso presenta en su interior cuatro pasos que constituyen un proceso que se puede reiniciar más de una vez, incluso una tercera. Para poner en evidencia cómo opera este proceso cuando debe reiniciarse, se expone a continuación, en la Figura 3, un ejemplo de los numerosos casos en que la organización retórica de la movida 2 Evolución del Caso funciona recursivamente. El texto se titula "Hepatotoxidad por acetaminófeno en un escolar con ingesta habitual de alcohol. Caso clínico" (CCMi-635). Antes de este 
fragmento, ya había sido presentado el paciente; se trata de un escolar de 12 años, de sexo masculino.

En el sexto párrafo del ejemplo, se observa que se reinicia el proceso de descripción de un estado inicial, con antecedentes del estado clínico y paraclínico del paciente. Finalmente, dado que los resultados expuestos (octavo párrafo) son favorables, la movida 2 finaliza y se presenta el epílogo de la historia, con el paso de la movida 3, la que, como veremos en los apartados siguientes, no aparece con la misma regularidad en todos los Casos Clínicos de las distintas especialidades.

\subsection{La organización retórica de la macromovida Relato del Caso a través de las especialidades médicas}

En conformidad con el último objetivo de este estudio, el Gráfico 2 da cuenta de la frecuencia de aparición de los pasos que conforman el Relato del Caso, a través de las nueve especialidades médicas en el Corpus CCM 2009. Dado que los propósitos comunicativos de los pasos contribuyen al propósito de las movidas, en el gráfico siguiente solo se incorporan los datos correspondientes a los ocho pasos retóricos.

Según lo indica el Gráfico 2, la frecuencia de ocurrencia tiende a ser relativamente homogénea en la mayoría de los pasos del Relato del Caso, a través de las especialidades. Este hecho se hace manifiesto en el traslapo que se produce entre algunas diferentes líneas del gráfico; aunque en él se pueden visualizar cinco líneas horizontales, en realidad los pasos retóricos incorporados en el gráfico son ocho. Se aprecia, eso sí, el leve alejamiento de esta tendencia de la especialidad de NP en los pasos Tratamiento del Caso (85\%) y Resultados (75\%). Esto significa que para la especialidad de Neuro-psiquiatría es menos relevante que para las demás detallar el desarrollo del tratamiento aplicado en el caso y reportar sus efectos en el paciente. 
El 28 de junio de 1997 ingresó al Servicio de Urgencia del Hospital de Castro (...), trasladado de urgencia desde el Hospital de Quellón, por haber presentado una crisis convulsiva tenicoclónica de etiología no precisada. Tenía el antecedente de cuadro respiratorio alto de una semana de evolución sin consulta médica, recibiendo acetaminófeno (Panadol) en tres dosis espaciadas de 500, 500 y $250 \mathrm{~V} / \mathrm{mg}$. durante el día anterior, indicadas y administradas por su madre. No se dispuso del antecedente alcohólico. En el examen físico: decaído, consciente y lúcido. Presión arterial: $85 / 50 \mathrm{~mm}$. $\mathrm{Hg}$, frecuencia cardiaca: $148 \times \mathrm{min}$, temperatura: $36,6^{\circ} \mathrm{C}$. Intensamente pálido, deshidratado, subietérico. Signos meníngeos negativos. Sin flapping. Examen segmentario normal.

Laboratorio de ingreso. Hematocrito; 22\%; Glóbulos blancos 13.500/mm3; Protrombina; 57\%; Tiempo de trombolastina parcial: $35 \mathrm{seg}$; Sodio $118 \mathrm{mEq} / \mathrm{L}$; Cloro $65 \mathrm{mEq} / \mathrm{L}$; Potasio: $5,2 \mathrm{mEq} / \mathrm{L}$; Glicemia: $77 \mathrm{mg} / \mathrm{dL}$; Uremia: $0,87 \mathrm{mg} / \mathrm{dL}$; Creatinina 1,08 mg/dL; Bilirrubina total; $1,36 \mathrm{mg} / \mathrm{dL}$; Liquido céfalo-raquídeo de aspecto y presión normal, estudio citológico y citoquímico normal.

Fue ingresado con la hipótesis diagnóstica de 1) Síndrome convulsivo ¿metabólico?, ¿infeccioso? Y 2$)$ P 2.3 Anemia.

Al ingreso presentó nuevo episodio convulsivo que se controló con diazepam endovenoso.

P 2.1

Inicio del Caso

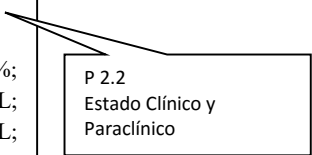

Estado Clínico y

Paraclínico

Diagnóstico

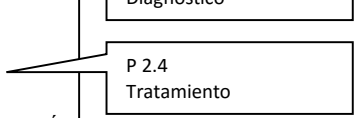

Dos horas después, hematemesis importante seguida de paro cardiorrespiratorio, del que se recuperó rápidamente. Recibió transfusión de sangre, vitamina K y corrección del desequilibrio electrolítico. En adelante, se mantuvo con hemodinámica estable, sin dificultad respiratoria, diuresis normal y sin nuevo $\widetilde{s}$ episodios convulsivos. En cuanto a conciencia, evolucionó hacia estado confusional. Los exámenes mostraron hematocrito en ascenso, mejoría lenta de las alteraciones electrolíticas, glicemia y función renal normales.

Veinticuatro horas después del ingreso, el paciente estaba febril, confuso y delirante. Transaminasad P 2.5 Resultados elevadas, aspartato aminotransferasa (GOT); $1.758 \mathrm{UI} / \mathrm{L}$ (rango; 7-21 Ul/L) y alanino aminotransferasa (GPT); $655 \mathrm{Ul} / \mathrm{L}$ (rango 7-26 'UI/L), los cuales aumentaron un día después a 5.250 y 1 `Q243 UI/L, respectivamente. Fosfatasas alcalinas normales. Bilirrubina total; $2,3 \mathrm{mg} / \mathrm{dL}$ de predominio directo. Protromhina $64 \%$.

Al tercer día se realizó una ecografía abdominal que informó hepatomegalia moderada e hígado graso sin otras alteraciones. En ese momento, al considerar que se estaba frente a una falla hepática grave con encefalopatía secundaria, se inició corticoterapia con prednisona $1 \mathrm{mg} / \mathrm{kg} /$ día por vía oral durante 48 horas, luego en dosis decrecientes hasta completar 10 días. El paciente evolucionó hasta la normalidad clínica y de laboratorio.

Al $11^{\circ}$ día, hematocrito de $34 \%$, transaminasas, bilirrubina y protrombina en rangos normales. Nueva ecografía abdominal permitió observar una disminución del tamaño hepático. La endoscopia digestiva alta informó esofagitis severa distal. La mejoría del paciente permitió su evaluación psico-social, confirmado por la madre, revelándose que mantenía en el último año una ingesta de chicha de manzana, vino o cerveza de 500 a $1.000 \mathrm{ml}$ casi diariamente, hasta 48 horas antes del ingreso; además, existía alcoholismo paterno y abandono escolar. Nunca había recibido una transfusión sanguínea ni tenía antecedentes familiares de hepatopatía. Alta en muy buenas condiciones al $19^{\circ}$ día. Un control quince días después, mostró normalidad clínica y de laboratorio.

Diez meses después se efectúa estudio de marcadores virales en Santiago (Hospital José Joaquín Aguirre), descartándose la existencia de una hepatitis viral crónica; los análisis para hepatitis B, C y E fueron negativos; anti VHA-IgM también negativo, pero sin embargo, el IgG fue positivo, evidenciando que este niño tuvo una hepatitis A en algún periodo antiguo de su vida, no pudiendo descartarse que el episodio agudo de casi un año antes haya podido ser una hepatitis A fulminante. Las pruebas de función hepática actuales son normales. (CCMI--635)

Figura 3. Ejemplo de dinamicidad de la movida 2 Evolución del Caso. 


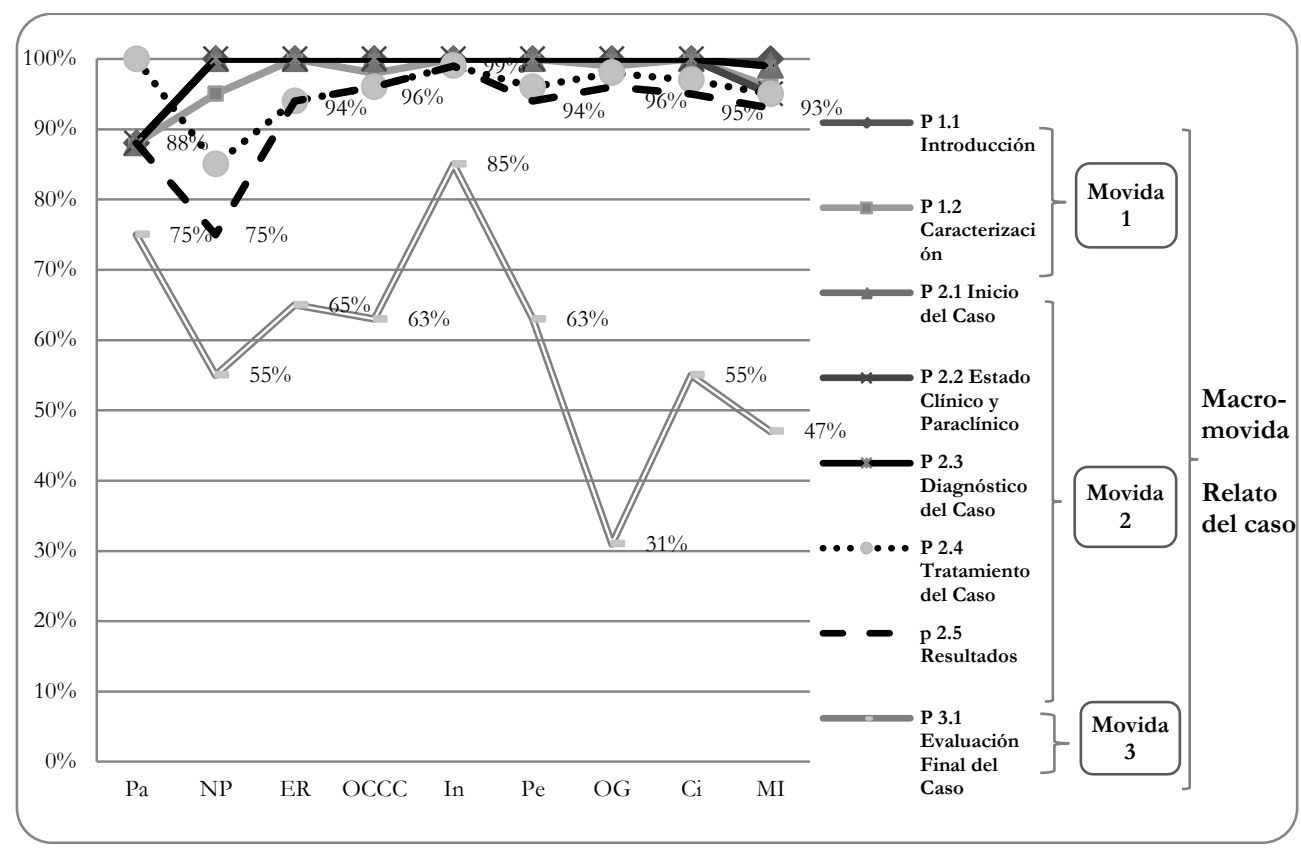

Gráfico 2. Pasos retóricos de macromovida Relato del Caso en las especialidades médicas del Corpus CCM-2009.

El paso 3.1 Evaluación Final del Caso, no obstante, constituye una excepción a la tendencia general recién señalada para las unidades retóricas. El propósito comunicativo de este paso es hacer un epílogo del relato, reportando una valoración del estado del paciente, realizada en un tiempo posterior a su tratamiento. Un ejemplo de Evaluación Final del Caso es el siguiente: "El paciente se mantiene en buenas condiciones generales y sin evidencias de recidiva tumoral un año después de realizado el diagnóstico" (CCER-58). En el ejemplo, es posible ver que este paso funciona a manera de epílogo, porque hace referencia a la situación que el sujeto presenta después de una mediana o gran distancia temporal del tiempo en fue tratado.

Pues bien, la tendencia general en el corpus es que el paso Evaluación Final del Caso ocurre con menor frecuencia, de lo que se deduce que es menos determinante para describir al género Caso Clínico. Si bien se mantiene como el menos frecuente en todas las especialidades, varía bastante su realización a través de ellas. De este modo, se advierte que en $\mathrm{Pa}(75 \%)$ e In $(85 \%)$ es muy frecuente que se haga referencia a qué ocurrió con el paciente en periodos posteriores de meses o años, lejanos a la crisis que relató el caso. En sentido contrario, se destaca que este paso presenta la más baja frecuencia en OG $(31 \%)$.

Considerando estos datos, puede suponerse que en algunas especialidades la variable tiempo determina prácticas profesionales distintas y que este hecho, connatural al quehacer de las especialidades, guardaría alguna relación con la mayor o menor 
presencia de un paso como Evaluación Final del Caso, en la macromovida Relato del Caso. Dicho de otro modo, para especialidades como la Parasitología y la Infectología, el propósito comunicativo realizado a través de este último paso retórico responde a una necesidad comunicativa que le sería propia por la naturaleza de su especialidad; esto es, el ejercicio profesional en ambas especialidades requiere hacer seguimiento a los pacientes después de una mediana o gran distancia temporal; por consiguiente, el Caso Clínico de Parasitología y de Infectología está más obligado a hacer referencia a esa situación. En sentido contrario, en la especialidad de Obstetricia y Ginecología, el factor tiempo juega un rol diferente, quizás porque los periodos de afección observados en algunas de sus áreas de trabajo son más breves y definidos, tales como los procesos de embarazo y parto, precisamente las áreas que más motivan la publicación de este género. En consecuencia, el discurso de la Obstetricia y Ginecología no necesitaría reportar el estado ulterior de los pacientes, porque no hay en la práctica profesional un seguimiento a mediano o largo plazo.

\section{CONCLUSIONES}

Los procedimientos seguidos en esta investigación y los resultados expuestos proveyeron evidencia empírica para realizar una descripción detallada de la organización retórica prototípica de la macromovida Relato del Caso, en el género Caso Clínico de la Medicina. Asimismo, permitieron comparar cómo se realiza dicha organización en las diversas especialidades médicas comprendidas en el corpus en estudio.

En cuanto a la organización del Relato del Caso para el género Caso Clínico, se concluye que esta macromovida, cuyo propósito comunicativo es narrar el desarrollo del caso, posee una organización retórica conformada por tres movidas retóricas cuyos propósitos se cumplen de manera sucesiva: presentar al paciente, exponer las fases del proceso experimentado por el paciente y, finalmente, referir el epílogo del relato. La cuantificación de las ocurrencias llevó a establecer que existen algunas movidas y pasos retóricos que presentan mayor o menor frecuencia de uso, lo que se relaciona con la mayor o menor obligatoriedad de ciertas unidades retóricas para el género. En este sentido, los pasos de las movidas 1 y 2, Establecimiento de situación inicial y Evolución del Caso, son, definitivamente, esenciales para la consecución de los propósitos de la macromovida en estudio y, por ende, para la configuración del genero Caso Clínico. La movida 3 y su paso retórico Evaluación final del Caso, en cambio, resulta opcional para el género.

Finalmente, si se compara la realización de la macromovida Relato del Caso en las 9 especialidades médicas a la que pertenecen los textos del corpus, se comprueba que en la mayoría de ellas la estabilidad de la macromovida Relato del Caso proviene principalmente de los propósitos comunicativos de la movida 1, Establecimiento de la situación, y de la movida 2 Evolución del Caso. En mucho menor grado, de la movida 
3, Epílogo. Esta última movida es particularmente importante en la realización del género en las especialidades de Parasitología e Infectología, pero es muy poco requerida en Obstetricia y Ginecología. Es evidente que las distintas prácticas profesionales -que a su vez responden a razones científicas- se ven reflejadas en la mayor o menor presencia de una movida del género.

Por otra parte, se pudo observar que la movida 2 Evolución del Caso es la que permite conseguir los propósitos centrales de la macromovida en estudio y, se podría suponer, los propósitos del género. Por otro lado, la Evolución del Caso es una movida dinámica, cuyos pasos operan cíclicamente, permitiendo, de este modo, dar cuenta del dinamismo de los procesos comunicados por el género. El examen de esta movida revela, además, que el Relato del Caso consigue sus propósitos comunicativos haciendo uso, principalmente, de las modalidades narrativa y descriptiva.

En síntesis, se comprobó que, en términos generales, la organización retórica de la macromovida Relato del Caso del género Caso Clínico tiende a ser relativamente homogénea a través de las especialidades. La explicación a esta regularidad es que las comunidades discursivas de la Medicina hacen uso de un sistema común de convenciones retóricas que se manifiestan en dicha estabilidad de su organización retórica funcional. Al mismo tiempo, una mirada más detallada de los datos revela que, en particular, existen algunas variaciones manifestadas en la relativa importancia de la movida 3 Epílogo con su paso retórico Evaluación Final del Caso, a través de las especialidades. Dichas variaciones pueden explicarse principalmente por la existencia de procedimientos profesionales propios de la naturaleza de la especialidad médica, los que se ven reflejados en la instanciación del género.

Entre las limitaciones que tuvo este estudio, se debe señalar que, al trabajar con un corpus que incluye solo textos publicados en revistas chilenas, las conclusiones descriptivas que se plantean podrían no representar al español de otras regiones. Entre sus fortalezas y proyecciones, está el que las unidades retóricas propuestas en este estudio pueden ser útiles para describir un segmento importante del Caso Clínico y para optimizar la producción escrita de discurso médico, en tanto explicita definiciones operacionales asociadas a los propósitos comunicativos de cada movida y paso. En este mismo sentido, los hallazgos de este estudio pueden servir como fuente de información para los profesores de lengua española en la universidad, pues provee un modelo de cómo se organiza más típicamente el Caso Clínico. 


\section{REFERENCIAS BIBLIOGRÁFICAS}

Adam, J. (1992). Les Textes: Types et Prototypes. Récit, Description, Argumentation, Explication et Dialogue. París: Nathan.

Adams-Smith, D. (1984). Medical Discourse: Aspects of Author's Comment. The ESP Journal, 3, 25-36.

Alcaraz, M. (2003). Las siglas del discurso biomédico escrito en inglés: Análisis y aplicaciones didácticas. The ESP, 23(1), 37-51.

Alcaraz, M. \& Salager-Meyer, F. (2003). La ciencia en pugna: Análisis retórico de la crítica en el discurso médico escrito en español (1930-1999). The ESPecialist, 24(1), 103-129.

Atkinson, D. (1992). The evolution of medical research writing from 1735 to 1985: The case of the "Edinburgh Medical Journal". Applied Linguistics, 13(4), 337-374.

Atkinson, P. (1995). Medical Talk and Medical Work. Londres: Sage.

Atkinson, P. (2004). The discursive construction of competence and responsibility in medical collegial talk. Communication \& Medicine, 1(1), 13-23.

Bart, N. \& Green, M. (2006). How to write a case report for publication. Journal of Chiropractic Medicine, 5(2), 72-82.

Bazerman, Ch. \& Paradis, J. (Eds.) (1991). Textual Dynamics of the Professions: Historical and Contemporary Studies of Writing in Professional Communities. Madison: The University of Wisconsin Press.

Berkenkotter, C. (2008). Patient Tales: Case Histories and the Uses of Narrative in Psychiatr. Columbia: University of South Carolina Press.

Bhatia, V. (1993). Analysing Genre: Language Use in Proffesional Settings. Londres: Longman.

Bhatia, V. (2004). Worlds of Written Discourse. Londres: Continuum

Biber, D. (1988). Variation Across Speech and Writing. Cambridge: Cambridge University Press.

Biber, D., Connor, U. \& Upton, T. (2007). Discourse on the Move: Using Corpus Analysis to Describe Discourse Structure. Amsterdam: Benjamins.

Brémond, C. (1966). La lógica de los posibles narrativos. En R. Barthes, A. Greimas, C. Brémond, J. Gritti, V. Morin, C. Metz, T. Todorov \& G. Genette (Eds.), Análisis Estructural del Relato (pp. 87-109). Buenos Aires: Editorial Tiempo Contemporáneo. 
Burdiles, G. (2012). Descripción de la organización retórica del género Caso Clínico de la Medicina a partir del Corpus CCM-2009. Tesis doctoral, Pontificia Universidad Católica de Valparaíso, Valparaíso, Chile.

Carey, J. (2006a). Significance of case reports in the advancement of medical scientific knowledge. American Journal of Medical Genetics, 140A, 2131-2134.

Carey, J. (2006b). A species not extinct: publication of case reports and scientific knowledge. American Journal of Medical Genetics, 140A, 801-803.

Cohen, H. (2006). How to write a patient case report. American Journal of Health-System Pharmacy, 63(19), 1888-1892.

Connor, U., Davis, K. \& De Rycker, T. (1995). Correctness and Clarity in Applying for Overseas Jobs: A Cross-Cultural Analysis of US and Flemish Applications. Text, 15(4), 457-475.

Crookes, G. (1986). Towards a validated analysis of scientific text structure. Applied Linguistics, 7(1), 57-70.

El Malik, A. \& Nesi, H. (2008). Publishing research in a second language: The case of Sudanese contributors to international medical journals. Journal of English for Academic Purposes, 7, 87-96.

Gérvas J., Pérez, M., Cuñat, V. \& Martínez, J. (2002). El caso clínico en medicina general. Aten Primaria, 30, 405-410.

Goic, A. (2002). La Revista Médica de Chile y la educación en Medicina. Revista Médica de Chile, 130(7), 719-722.

Gotti, M. (2003). Specialized Discourse: Linguistic Features and Changing Conventions. Berna: Peter Lang.

Hernández, R., Fernández, C. \& Baptista, P. (2010). Metodología de la Investigación. México: McGraw-Hill.

Hiltunen, T. (2006). Coming-to-know verbs in research articles in three academic disciplines. En M. Pérez-Llantada, R. Plo \& C. Neumann (Coords.), Actas de V Congreso Internacional AELFE (pp. 246-251). Zaragoza: Universidad de Zaragoza.

Holmes, R. (1997). Genre Analysis, and the Social Sciences: An Investigation of the Structure of Research Article Discussion Sections in Three Disciplines. English for Specific Purposes, 16(4), 321-337. 
Ibáñez, R. (2008). El texto disciplinar y el acceso al conocimiento desde el análisis del género: ¿Regulación del conocimiento o persuasión? En G. Parodi (Ed.), Géneros Académicos y Géneros Profesionales: Accesos Discursivos para Saber y Hacer (pp. 219-246). Valparaíso: Ediciones Universitarias de Valparaíso.

Jefferson, T. (2008). More cases, doctor? Yes please! Cases Journal, 1, 38-40

Jenicek, M. (2001). Clinical Case Reporting in Evidenced-Based Medicine. Londres: Arnold.

Kanoksilapatham, B. (2005). Rhetorical structure of biochemistry research articles. English for Specific Purposes, 24, 269-292.

Kanoksilapatham, B. (2007a). Rhetorical moves in biochemistry research articles. En D. Biber, U. Connor \& T. Upton (Eds.), Discourse on the Move. Using Corpus Analysis to Describe Discourse Structure (pp. 73-119). Amsterdam: John Benjamins.

Kanoksilapatham, B. (2007b). Introduction to move analysis. En D. Biber, U. Connor \& T. Upton (Eds.), Discourse on the Move. Using Corpus Analysis to Describe Discourse Structure (pp. 23-41). Amsterdam: John Benjamins.

Kanoksilapatham, B. (2007c). Introduction to move analysis. En D. Biber, U. Connor \& T. Upton (Eds.), Discourse on the move. Using corpus analysis to describe discourse structure (pp. 23-41). Amsterdam: John Benjamins.

Khan, K. \& Thompson, P. (2002). A proposal for writing and appraising case reports. International Journal of Obstetrics and Gynaecology, 109(8), 849-851.

Li, L. \& Ge, G. (2009). Genre analysis: Structural and linguistic evolution of the English-medium medical research article (1985-2004). English for Specific Purposes, 28, 93-104.

Mertens, D. (2005). Research and Evaluation in Education and Psychology: Integrating Diversity with Quantitative, Qualitative, and Mixed Methods. Thousand Oaks: Sage.

Morales, O. (2010). Los géneros escritos de la odontología hispanoamericana. Estructura retórica y estrategias de atenuación en articulos de investigación, casos clínicos y articulos de revisión. Tesis doctoral, Universidad Pompeu Fabra, Barcelona, España.

Morales, O., Cassany, D., Marín, E. \& González, C. (2007). El discurso escrito de la Odontología: Análisis retórico discursivo de casos clínicos hispanos (19992005). Revista MedULA, 16(2), 75-82.

Nwogu, K. (1995). Structuring scientific discourse using the "given-new" perspective. English Teaching Forum, 33(4), 22-28.

Nwogu, K. (1997). The medical research paper: Structure and function. English for Specific Purposes, 16(2), 119-138. 
Oliver, S. (2004). Análisis contrastivo español/ inglés de la atenuación retórica en el discurso médico. El artículo de investigación y el caso clínico. Tesis doctoral, Universidad Pompeu Fabra, Barcelona, España.

Parodi, G. (2008a). La organización retórica del género Manual: ¿Una “colonia encadenada"? En G. Parodi (Ed.) Géneros Académicos y Géneros Profesionales: Accesos Discursivos para Saber y Hacer (pp. 169-198). Valparaíso: Ediciones Universitarias de Valparaíso.

Parodi, G. (2008b). El género Manual y su organización retórica en cuatro disciplinas científicas: Entre la abstracción y la concreción. En G. Parodi (Ed.) Géneros Académicos y Géneros Profesionales: Accesos Discursivos para Saber y Hacer (pp. 199218). Valparaíso: Ediciones Universitarias de Valparaíso.

Parodi, G., Venegas, R., Ibáñez, R. \& Gutiérrez, R. (2008). Géneros del discurso en el Corpus PUCV-2006: criterios, definiciones y ejemplos. En G. Parodi (Ed.) Géneros Académicos y Géneros Profesionales: Accesos Discursivos para Saber y Hacer (pp. 39-74). Valparaíso: Ediciones Universitarias de Valparaíso.

Parodi, G., Boudon, E. \& Julio, C. (2014). La organización retórica del género Manual de Economía: Un discurso en tránsito disciplinar. Revista de Lingüistica Teórica y Aplicada, 52(2), 133-163.

Pertuzé, J. (2006). Criterios para publicar casos clínicos. Revista Chilena de Enfermedades Respiratorias, 22, 105-107.

Reyes, C. \& Llanos, G. (2002). La alegría de publicar. El informe de un caso clínico. Colombia Médica, 33(4), 198-199.

Salager-Meyer, F. (1992). A text-type and move analysis of verb tense and modality distribution in medical English abstracts. English for Specific Purposes, 11(2), 93115.

Salager-Meyer, F. (1994). Hedges and textual communicative function in medical english written discourse. English for Specific Purposes, 13(2), 149-170.

Salager-Meyer, F. \& Alcaraz, M. (2003). Academic criticism in Spanish medical discourse: a cross-generic approach. International Journal of Applied Linguistics, 13(1), 96-114.

Samraj, B. (2002). Introductions in research articles: Variations across disciplines. English for Specific Purposes, 21, 1-17.

Serrano, E. (2010). El caso clínico de lo clásico a lo cierto. Butlletí, 28(04) [en línea]. Disponible en: http://creativecommons.org/licenses/by-nc-nd/2.5/es/. 
Skelton, J. (1994). Analysis of the structure of original research papers: an aid to writing original papers for publication. British Journal of General Practice, 44, 455-459.

Skelton, J. (1997). The representation of truth in academic medical writing. Applied Linguistics, 18(2), 121-140.

Swales, J. (1981). Aspects of Article Introductions. Birmingham: Aston University Languages Study.

Swales, J. (1990). Genre Analysis. English in Academia and Research Settings. Cambridge: Cambridge University Press.

Swales, J. (2004). Genre Analysis: Research Genres. Explorations and Applications. Cambridge: Cambridge University Press.

Taavitsainen, I. \& Pahta, P. (2000). Conventions of professional writing: The medical case report in a historical perspective. Journal of English Linguistics, 28, 60-76.

Tashakkori, A. \& Teddlie, C. (2003). Handbook of Mixed Methods in Social \& Behavioral Research. Thousand Oaks: Sage.

Uribarri, I. (2007). La descripción científica y el caso clínico. Revista de Neurocirugía, 9(2), 56-63.

Valladolid, A. \& Pérez, R. (2005). Requisitos de uniformidad para manuscritos remitidos a revistas biomédicas: Redacción y edición de la publicación biomédica Comité Internacional de Editores de Revistas Médicas (ICMJE). Farmacia Hospitalaria, 29(3), 191-208.

Varttala, T. (2001). Hedging in scientifically oriented discourse. Exploring variation according to discipline and intended audience. Tesis doctoral, Universidad de Tampere, Tampere, Finlandia.

Vandenbroucke, J. (2001). In defense of case reports and case series. Annals of Internal Medicine, 134, 330-334.

Venegas, R. (2014). El trabajo final de Grado de Licenciatura: Caracterización discursiva y andamiaje didáctico. Conferencia presentada en el 3er Congreso Nacional de Pedagogía Universidad de Los Lagos, Osorno, Chile

Xunta de Galicia (2013). Guía de Recomendaciones para la Gestión de Eventos Centinela y Evento Adversos Graves. Santiago de Compostela: Servicio Galego de Saúde 